\title{
Understanding the benign nature of SIV infection in natural hosts
}

\author{
Guido Silvestri, ${ }^{1,2}$ Mirko Paiardini, ${ }^{1}$ Ivona Pandrea, ${ }^{3}$ Michael M. Lederman, ${ }^{4}$ and Donald L. Sodora ${ }^{5}$ \\ ${ }^{1}$ Department of Pathology and Laboratory Medicine, University of Pennsylvania, Philadelphia, Pennsylvania, USA. ${ }^{2}$ Yerkes National Primate Research Center, \\ Emory University, Atlanta, Georgia, USA. ${ }^{3}$ Tulane National Primate Research Center, Tulane Health Sciences Center, Tulane University, Covington, \\ Louisiana, USA. ${ }^{4}$ Department of Medicine, Case Western Reserve University, Cleveland, Ohio, USA. ${ }^{5}$ Department of Medicine, \\ University of Texas Southwestern Medical Center, Dallas, Texas, USA.
}

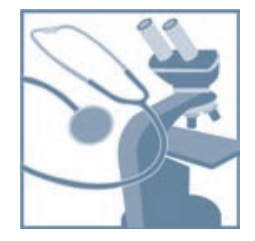

In striking contrast to HIV infection, natural SIV infection of African nonhuman primates is asymptomatic and usually does not induce significant $\mathrm{CD} 4^{+} \mathrm{T}$ cell depletion despite high levels of virus replication. Recently, significant progress has been made in understanding the mechanisms underlying the remarkable difference in infection outcome between natural and nonnatural HIV/ SIV hosts. These advances include the identification of limited immune activation as a key factor protecting natural SIV hosts from AIDS and the discovery of low CC chemokine receptor 5 expression on $\mathrm{CD}^{+} \mathrm{T}$ cells as a specific and consistent immunologic feature in these animals. Further elucidation of the pathways by which the differences in immune activation between natural and nonnatural hosts are manifest holds promise for the design of novel therapeutic approaches to HIV infection.

Simian immunodeficiency viruses (SIVs) are primate lentiviruses that infect numerous nonhuman primate (NHP) species in Africa. Two of these viruses, SIVcpz from chimpanzees (Pantroglodytes) and SIVsmm from sooty mangabeys (SMs, Cercocebus atys), have crossed species barriers on multiple occasions, resulting in the generation of HIV-1 and HIV-2, respectively (1). Other African monkey species that are naturally infected with SIV include African green monkeys (AGMs), mandrills, sun-tailed monkeys, L'hoest monkeys, Sykes monkeys, and numerous others (2). A key feature of SIV infection of natural hosts is the lack of clinical disease, with the animals living an apparently normal lifespan (reviewed in refs. 2-7). Instances of AIDS-like diseases in naturally SIV-infected NHPs are exceedingly rare, with only a few cases of AIDS described in natural hosts (reviewed in ref. 2). In sharp contrast, experimental SIV infection of non-natural host Asian monkey species, such as rhesus macaques (RMs; Macaca mulatta) results in the development of illness similar to that described in AIDS patients (simian AIDS). At present, the exact events that make SIV infection generally nonpathogenic in natural host monkey species and those that induce immunodeficiency in nonnatural hosts (including humans, who have only recently become a host species for HIV) are still unclear. It is reasonable to propose that a further elucidation of the mechanisms underlying the benign course of SIV infection in its "natural" hosts will likely provide important insights into the determinants of immune deficiency in HIV-infected humans. Thus, there is an emphasis on identifying the differences between natural SIV infection and the typically pathogenic infections of humans and RMs, with the implicit assumption that phenomena observed in both settings are less important for disease progression than those observed in pathogenic infections only. In this review, we

Nonstandard abbreviations used: AGM, African green monkey; CCR5, CC chemokine receptor 5; CXCR4, CXC chemokine receptor 4; LTNP, long-term nonprogressor; MALT, mucosa-associated lymphoid tissue; NHP, nonhuman primate; RM, rhesus macaque; SM, sooty mangabey.

Conflict of interest: The authors have declared that no conflict of interest exists. Citation for this article: J. Clin. Invest. 117:3148-3154 (2007). doi:10.1172/JCI33034. will summarize a series of recent advances in our understanding of the benign nature of SIV infection in natural hosts and discuss the implications of these findings for HIV/AIDS pathogenesis, therapy, and vaccines.

\section{Basic features of natural SIV infection}

SIV infections of natural hosts are typically nonpathogenic and characterized, in the vast majority of cases, by sustained preservation of peripheral CD4 ${ }^{+} \mathrm{T}$ cell counts $(8-17)$. $\mathrm{CD}^{+} \mathrm{T}$ cell preservation is sometimes seen in HIV-infected individuals termed long-term nonprogressors (LTNPs), yet LTNPs characteristically control HIV replication, while naturally SIV-infected NHPs sustain levels of viremia that are as high or often even higher than those observed in HIV-infected individuals with disease progression (8-18). Figure 1 summarizes the key differences and similarities between nonpathogenic SIV infection of natural hosts and LTNPs. In a recent survey of 110 naturally SIV-infected SMs housed at the Yerkes National Primate Research Center, we found a mean viral load of 170,000 $\pm 205,000$ copies/ml of plasma and a mean $\mathrm{CD}^{+}$ $\mathrm{T}$ cell count of $1,067 \pm 589$ cells $/ \mathrm{mm}^{3}(19,20)$. This typical phenotype of natural SIV hosts - high $\mathrm{CD}^{+} \mathrm{T}$ cell counts despite high viral loads - is very different from the observation that in both HIV-infected individuals and experimentally SIVmac-infected RMs, high levels of virus replication predict faster disease progression (21-23). On the contrary, difference in the level of viremia between SMs does not appear to impact the clinical course. That virus replication is continuously high during nonpathogenic SIV infection of natural hosts is a consistent finding, the importance of which cannot be overstated. The first fundamental implication is that the evolutionary coadaptation between lentiviruses and their natural primate hosts that permits peaceful coexistence does not appear to involve better immune control of virus replication. Indeed, the level of SIV-specific T cell responses in SMs is comparable to that in RMs and is neither stronger nor broader than what has been found during pathogenic HIV/SIV infections (24-26). Furthermore, depletion of $\mathrm{CD}^{+} \mathrm{T}$ cells in SMs was found to be followed by minor changes in SIV replication in the only published 


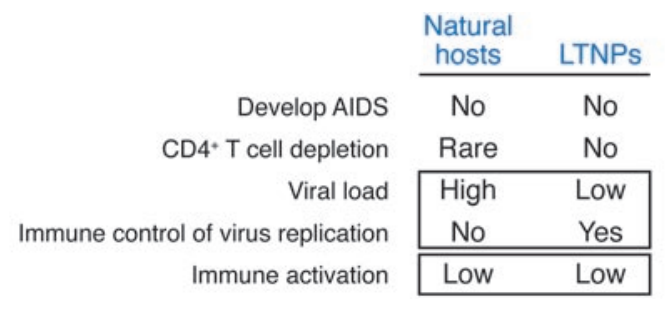

Figure 1

Similarities and differences between nonpathogenic SIV infection of natural hosts and HIV infection of LTNPs.

study at this writing (27). From a vaccine perspective, the fact that the AIDS resistance of natural SIV hosts appears to be independent of effective control of virus replication emphasizes the tremendous challenge of artificially inducing, via immunization, a type of protective immunity that has not been selected for through many thousand years of evolutionary pressure posed by lentiviruses on the primate immune system. The second fundamental implication of the high-level viremia in natural SIV infections is that we must reevaluate the concept that the direct HIV-mediated killing of infected $\mathrm{CD}^{+} \mathrm{T}$ cells is both necessary and sufficient to cause progression to AIDS in HIV-infected individuals. Instead, a more consistent model may be that the HIV-associated immunodeficiency is a complex phenomenon initially triggered by the virus but ultimately related to the nature of the host response to infection (28-31).

\section{Natural SIV infections are characterized by low immune activation}

An important common feature of SIV infection in natural hosts is that these animals consistently show levels of immune activation that are significantly lower than those observed in HIV-infected humans or SIV-infected RMs (13, 14, 16-20, 32-34). In SMs, perhaps the most well-characterized model of natural SIV hosts, it was shown that chronic SIV infection is associated with (a) low levels of $\mathrm{CD}^{+}$and $\mathrm{CD}^{+} \mathrm{T}$ cell activation and proliferation in both peripheral blood and lymph nodes (14, 16-18); (b) no signs of increased levels of T cell apoptosis either in vivo or in vitro (17, $35-36)$; (c) absence of the cell-cycle dysregulation associated with pathogenic HIV/SIV infections (37-39); and (d) preserved function of the $\mathrm{T}$ cell regenerative compartment, i.e., bone marrow, thymus, and lymph nodes $(17,40)$. In addition, a strong inverse correlation was found between the level of circulating effector $T$ cells (reflective of general $\mathrm{T}$ cell activation) and $\mathrm{CD}^{+} \mathrm{T}$ cell counts in naturally SIV-infected SMs (19), suggesting that the establishment of a higher set-point level of immune activation has a negative effect on $\mathrm{CD}^{+} \mathrm{T}$ cell homeostasis even in the setting of a nonpathogenic infection. Importantly, the levels of $\mathrm{T}$ cell activation and proliferation observed during the acute phase of experimental SIVsmm infection of SMs are blunted compared with those observed during pathogenic SIVmac infection of RMs, with a lower peak and a more pronounced postpeak decline of immune activation (40-42). Taken together, these findings indicate that limited immune activation is a consistent and distinct feature of natural SIV infection when compared with all known models of pathogenic HIV/SIV infection. It should be noted that this low-level immune activation in natural hosts does not appear to represent ignorance of or tolerance to SIV antigens, as SIV-specific T cells are present in naturally
SIV-infected SMs $(25,26)$ and appear to exert some immune pressure on the virus (43). At least in SMs, however, the magnitude of these SIV-specific T cell responses is lower than the HIV-specific $\mathrm{T}$ cell responses seen in humans (25).

With these findings in mind, we and others have proposed that it is the low level of immune activation that protects natural SIV hosts from $\mathrm{CD}^{+} \mathrm{T}$ cell depletion and $\operatorname{AIDS}(2,6)$. This postulate is consistent with the hypothesis that, during pathogenic HIV/ SIV infections, generalized immune activation is an important driver of disease progression (28-31). The pathogenic impact of the hyperimmune activation seen in nonnatural hosts has been emphasized by several important observations, including the fact that, in HIV-infected individuals, levels of $\mathrm{T}$ cell activation are a very strong predictor of disease progression (44-46).

Intense studies are currently in progress to identify the mechanisms that permit natural SIV hosts to maintain low levels of immune activation during both acute and chronic infection despite continuous high-level virus replication. In experimentally SIVagm-infected AGMs, an early increase in numbers of Tregs is paralleled by an increase in TGF- $\beta$ and IL-10 levels in the plasma during the acute phase of infection (32). This antiinflammatory milieu may explain the low and transitory $\mathrm{T}$ cell immune activation in this natural host. Normal Treg development after infection has also been observed in SMs $(34,47)$. Collectively, these data suggest that Treg responses may be instrumental in attenuating the immune activation during natural SIV infections. Yet the immune activation that characterizes pathogenic HIV/SIV infection is too extensive to be restricted to a few antigenic specificities. Thus, additional mechanisms have been proposed to be responsible for the low set-point level of immune activation in natural SIV hosts that may act at a number of different molecular and cellular levels, including the dendritic cell-T cell interface, the proinflammatory cytokine response, the regulation of $\mathrm{T}$ cell homing to inflamed tissues, and maintenance of the balance of distinct $\mathrm{CD}^{+} \mathrm{T}$ cell subsets (Th1, Th2, Th17), among various others. Interestingly, a recent study identified a potential role for the nef gene products of various SIVs (but not of HIV) in protecting against chronic immune activation and $\mathrm{CD}^{+} \mathrm{T}$ cell depletion during natural SIV infection by downregulating the expression of the CD3-TCR complex from the surface of infected CD4 ${ }^{+} \mathrm{T}$ cells (48). Future experiments in which natural SIV hosts (i.e., SMs and/or AGMs) are inoculated with recombinant SIVs expressing nefs that, similar to HIV-1 nef, have lost the ability to downmodulate expression of the CD3-TCR complex may be useful for the in vivo assessment of the hypothesis that SIVnef-mediated downregulation of CD3-TCR protects natural SIV hosts from disease progression.

\section{Mucosal CD4 ${ }^{+} \mathrm{T}$ cells are depleted in natural SIV hosts}

A series of recent studies showed that pathogenic HIV infection of humans and SIV infection of RMs are characterized by an early, severe, and largely irreversible depletion of mucosal CD $4^{+} \mathrm{CCR} 5^{+}$ memory T cells (where CCR5 is CC chemokine receptor 5) (49-54). Based on these studies, a model of AIDS pathogenesis has been formulated whereby the selective depletion of memory $\mathrm{CD}^{+}$ $\mathrm{T}$ cells from mucosal tissues during acute HIV infection is a key determinant of disease progression, as it results in a severe impairment of mucosal immunity (55-58). Recently, a mechanistic link was proposed between this early defect in mucosal immunity and the establishment of the typically high levels of immune activation seen during pathogenic HIV/SIV infections (59). Breakdown 
HIV-infected humans

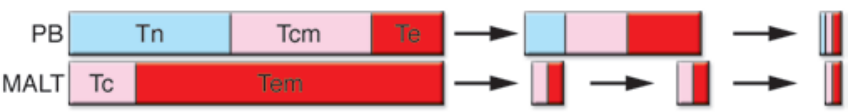

SIV-infected SMs "CD4-high"
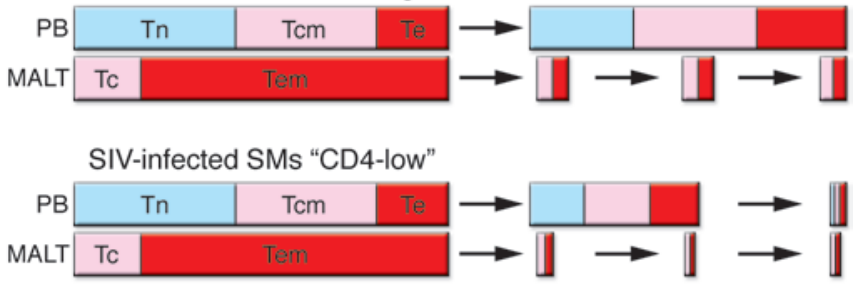

Time from primary infection

Figure 2

Dynamics of the CD4+ $\mathrm{T}$ cell pools during pathogenic and nonpathogenic lentiviral infections. This figure represents the changes induced by HIV/SIV infection in the size of the pools of naive (Tn, blue), memory (Tcm, pink), and effector (Tem, red) $\mathrm{CD}^{+}{ }^{+} \mathrm{T}$ cell subsets in both peripheral blood (PB) and MALT. The top row shows how HIV infection is associated with rapid decline of the mucosal $C D 4^{+} \mathrm{T}$ cells and much slower, but eventually very severe, depletion of the circulating pool of $\mathrm{CD}^{+}{ }^{+}$cells (this latter event is associated with progression to AIDS). The middle row shows how nonpathogenic SIV infection of SMs is associated, in approximately $90 \%$ of cases, with preservation of the peripheral pool of $\mathrm{CD}^{+} \mathrm{T}$ cells but a rapid depletion of the same cells in mucosal tissues. The bottom row shows how a minority of SIVinfected SMs experience a severe depletion of CD4+ $\mathrm{T}$ cells in both peripheral blood and MALT — and yet do not exhibit any clinical signs of AIDS. The term "CD4-low" refers to the small subset $(\sim 3 \%-5 \%)$ of SIV-infected SMs with CD4+ $\mathrm{T}$ cell counts below 200 cells $/ \mathrm{mm}^{2}$.

of the mucosal barrier as a result of the massive depletion of $\mathrm{CD} 4^{+}$ $\mathrm{T}$ cells favors the translocation of microbial products (e.g., LPS and others) from the intestinal lumen to the systemic circulation, causing broad activation of the immune system via their binding to certain TLRs $(59,60)$.

Interestingly, an early, severe, and persistent depletion of mucosal $\mathrm{CD}^{+} \mathrm{T}$ cells has now also been observed during nonpathogenic SIV infection of the natural hosts - SMs and AGMs (42, 61 ). The kinetics of this early depletion are remarkably similar in both species to those observed during highly pathogenic SIVmac239 infection of RMs $(42,61)$. It should be noted, however, that in contrast to SIV-infected RMs (in whom mucosal CD4 ${ }^{+} \mathrm{T}$ cell depletion becomes increasingly more severe as disease progresses to AIDS), the early mucosal $\mathrm{CD}^{+} \mathrm{T}$ cell depletion of natural hosts either does not progress further after reaching a stable plateau (in SMs) or is followed by a significant recovery of these cells (in AGMs) $(42,61)$. The realization that the homeostasis of mucosa-associated lymphoid tissue (MALT) $\mathrm{CD}^{+} \mathrm{T}$ cells but not blood $\mathrm{CD}^{+} \mathrm{T}$ cells is, in fact, disrupted during nonpathogenic SIV infection indicates that the impact of SIV on the dynamics of the $\mathrm{CD}^{+} \mathrm{T}$ cell pool in natural hosts is more complex than previously appreciated (Figure 2). Importantly, the presence of an early and severe depletion of mucosal CD4 ${ }^{+} \mathrm{T}$ cells in natural SIV hosts who remain asymptomatic means that the loss of MALT $\mathrm{CD} 4^{+} \mathrm{T}$ cells cannot be sufficient for the development of AIDS but indicates instead that other factors are required to induce mucosal and systemic immune dysfunction during a primate lentiviral infection. Consistent with the hypothesis that natural hosts maintain normal integrity of their mucosal barrier despite the severe loss of mucosal CD4 ${ }^{+} \mathrm{T}$ cells is the important observation that, in naturally SIV-infected SMs and experimentally SIVinfected AGMs, plasma levels of LPS are not elevated, remaining at levels similar to those found in uninfected animals $(60,61)$.

Collectively, these studies of mucosal immunity in SIV-infected SMs and AGMs raise a key question: what mechanisms protect natural SIV hosts from progressing to AIDS in the setting of a profound depletion of mucosal $\mathrm{CD}^{+} \mathrm{T}$ cells? One obvious possibility is that natural SIV hosts have evolved to be less dependent on $\mathrm{CD}^{+} \mathrm{T}$ cells to maintain the overall function of the mucosal immune system - a possibility supported by the fact that normal AGMs show very low levels of CD4 ${ }^{+} \mathrm{T}$ cells even before infection (14). This relative "CD4 independence" could be the result of evolutionary pressure placed upon these animals by lentiviral infections that caused them to progressively adapt to tolerate levels of mucosal $\mathrm{CD}^{+} \mathrm{T}$ cell depletion that would be associated with disease progression in humans or RMs. An alternative possibility is that additional factors, in particular the lack of local immune activation, protect the $\mathrm{CD} 4^{+} \mathrm{T}$ cell-depleted mucosae of natural SIV hosts from losing their barrier function. This hypothesis is consistent with the observation that, in both SMs and AGMs, the level of mucosal immune activation (measured as the fraction of T cells expressing markers of activation and proliferation) during both acute and chronic SIV infection is markedly lower than in SIV-infected RMs $(42,61)$. An additional possibility is that the early mucosal $\mathrm{CD} 4^{+} \mathrm{T}$ cell depletion observed in both pathogenic and nonpathogenic lentiviral infections is not sufficient to initiate the events leading to AIDS (as it can be tolerated without clinical manifestations in natural hosts) but rather serves as a key mechanism to promote virus dissemination and establish chronic infection by generating a large and diffuse pool of infected cells during acute infection.

\section{Extreme $\mathrm{CD}^{+} \mathrm{T}$ cell depletion in SMs infected with dual-tropic SIV}

While the majority of naturally SIV-infected SMs maintain normal CD4 ${ }^{+} \mathrm{T}$ cell counts throughout the infection, a subset $(10 \%-$ $15 \%$ ) of these animals experience a significant depletion (i.e., to less than 500 cells $/ \mathrm{mm}^{2}$ of circulating CD4 ${ }^{+} \mathrm{T}$ cells; ref. 19; Figure 2). Intriguingly, two naturally SIV-infected SMs have been discovered with levels of $\mathrm{CD}^{+} \mathrm{T}$ cells below 50 cells $/ \mathrm{mm}^{2}$, i.e., a CD $4^{+} \mathrm{T}$ cell count that would place HIV-infected humans or SIV-infected RMs at high risk of opportunistic infections and death (19). Important insights into the pathogenesis of this unusual "CD4low" phenotype of a minority of SIV-infected SMs were provided by a recent study that revealed that extreme (i.e., less than $0.2 \%$ of residual $\mathrm{CD}^{+} \mathrm{T}$ cells) and generalized (i.e., observed in blood, lymph nodes, and mucosal tissues) $\mathrm{CD}^{+} \mathrm{T}$ cell depletion can be observed in SIV-infected SMs in association with the emergence of SIV strains with an expanded coreceptor tropism (62). The ability to use CXC chemokine receptor 4 (CXCR4) as well as CCR5 as a coreceptor for virus entry is highly uncommon in SIV-infected SMs as well as other natural hosts (with only SIVagm.sab strains using CXCR4), as primary SIV isolates have previously been found to use mainly CCR5 as a coreceptor for host cell entry $(63,64)$. It is interesting that the emergence of dual-tropic CXCR4-tropic viruses results in an almost complete depletion of $\mathrm{CD}^{+} \mathrm{T}$ cells, as a similar phenomenon has been observed in HIV-infected patients 

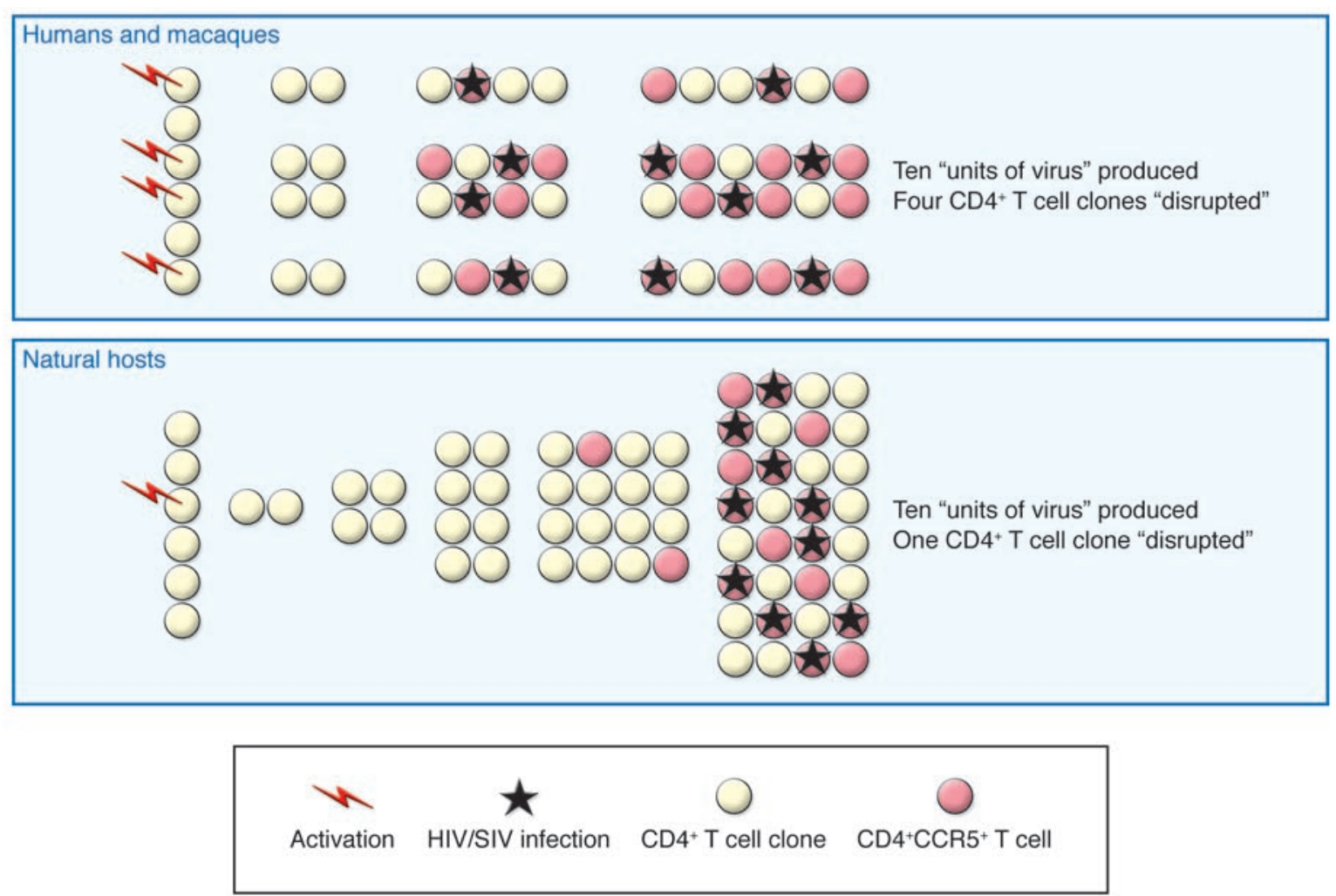

\section{Figure 3}

Potential synergy between low immune activation and reduced CCR5 expression in preserving CD4+ T cell homeostasis in SIV-infected SMs despite high virus replication. This figure shows how, in SIV-infected SMs (natural hosts), low immune activation, expressed as the fraction of $\mathrm{CD} 4^{+} \mathrm{T}$ cell clones that undergo activation at any given time, may act in concert with reduced and/or delayed expression of CCR5 in promoting a steady state where immune system homeostasis is preserved despite high virus replication. The top row shows how in HIV-infected humans and SIV-infected RMs, presence of a high fraction of activated CD4 ${ }^{+} \mathrm{T}$ cell clones results in the rapid accumulation of $\mathrm{CD} 4^{+} \mathrm{T}$ cells expressing CCR5 that are infected and killed by the virus, resulting in the disruption of the homeostasis of these activated clones. In SIV-infected SMs (bottom row), a smaller fraction of CD4+ T cells clones are activated, but a delayed and reduced expression of CCR5 on these cells may allow for their accumulation, resulting in an equally high level of virus replication when CCR5 is finally expressed. In this case, however, the homeostasis of fewer $\mathrm{CD} 4^{+} \mathrm{T}$ cells clones is disrupted at any given time.

$(65,66)$. What is remarkably different, however, is that these $\mathrm{CD}^{+}{ }^{+} \mathrm{T}$ cell-depleted SIV-infected SMs have remained completely asymptomatic for a prolonged period (i.e, more than 5 years) (62). This fascinating observation suggests the possibility that the absence of chronic immune activation (as these "CD4-low" SIVinfected SMs display the typical phenotype of natural SIV hosts characterized by low levels of $\mathrm{T}$ cell activation and proliferation) is sufficient to protect these animals from developing the clinical manifestations of AIDS even when their $\mathrm{CD}^{+} \mathrm{T}$ cells are almost completely lost. Conceivably, lower levels of immune activation may favor the preservation of the number and/or function of other immune cell types (including $\mathrm{CD}^{+} \mathrm{T}$ cells, $\gamma \delta \mathrm{T}$ cells, NK cells, macrophages, etc.) such that their functional integrity may compensate for the profound $\mathrm{CD}^{+} \mathrm{T}$ cell depletion (62). In this regard, it should be noted that during chronic, progressive HIV infection, high levels of immune activation are associated with decreased function of many immune cell types (67-72).

\section{Low CCR5 expression on CD4 ${ }^{+} \mathrm{T}$ cells of natural hosts}

As discussed above, the level of immune activation is clearly a major and consistent difference between natural, nonpathogenic and nonnatural, pathogenic HIV/SIV infections. Another striking immunologic difference that we have identified recently is the level of CCR5 expression on $\mathrm{CD} 4^{+} \mathrm{T}$ cells, which is significantly lower in natural SIV hosts (73). Low levels of CD $4^{+} \mathrm{CCR} 5^{+} \mathrm{T}$ cells were found in the blood, lymph nodes, and mucosal tissues of natural SIV hosts and were observed across several species including SMs, AGMs, chimpanzees, mandrills, and solatus monkeys, indicating a striking evolutionary convergence of this characteristic phenotype (73). Importantly, the low-level CCR5 expression is specific for $\mathrm{CD}^{+} \mathrm{T}$ cells (and, to a lesser extent, for monocytes/macrophages), as $\mathrm{CD}^{+} \mathrm{T}$ cells in these hosts express levels of CCR5 comparable to those observed in nonnatural hosts (73). While the low expression of CCR5 on $\mathrm{CD}^{+} \mathrm{T}$ cells is a strikingly clear finding, it does not protect natural SIV hosts from infection with CCR5-tropic virus and subsequent high-level virus replication that, similar to pathogenic infections, occurs predominantly in activated $\mathrm{CD}^{+} \mathrm{T}$ cells $(17,41)$. Thus, the effects of the low CCR5 expression on CD $4^{+}$ $\mathrm{T}$ cells of natural hosts may be more complex; perhaps reduced homing of activated $\mathrm{CD} 4^{+} \mathrm{CCR} 5^{+} \mathrm{T}$ cells to inflamed tissues and diminished signaling in response to these chemokine ligands may blunt the cycles of HIV/SIV-associated immune activation (73). 
An attractive alternative hypothesis is that, in natural SIV hosts, expression of CCR5 is restricted to $\mathrm{CD}^{+} \mathrm{T}$ cells that, after having encountered their cognate antigen, are in a more advanced stage of activation and/or differentiation to effectors (see Figure 3). As such, restricted and delayed expression of CCR5 on activated CD $4^{+}$ $\mathrm{T}$ cells may act in concert with a state of attenuated immune activation to preserve the homeostasis of the pool of "resting" naive and memory $\mathrm{CD}^{+} \mathrm{T}$ cells while supporting similarly high levels of virus production. During any immune response, the majority of activated $\mathrm{CD}^{+}{ }^{+} \mathrm{T}$ cells are likely to die as a consequence of activation-induced cell death (AICD) (74). Therefore, limiting virus replication - by virtue of selective CCR5 expression - to a subset of more "expendable" highly activated effector $\mathrm{CD} 4^{+} \mathrm{T}$ cells that are likely destined for AICD (regardless of their SIV infection status) could be a very elegant mechanism for the host immune system to both support and tolerate high virus replication and chronic destruction of activated $\mathrm{CD}^{+} \mathrm{T}$ cells (Figure 3 ).

\section{Evolutionary considerations}

Scientists working in this field generally assume that the coadaptation of primate lentiviruses and their natural hosts has slowly coevolved over many thousands of years of coexistence to result in the nonpathogenic infection phenotype that has permitted survival of both (2-7). As the coevolution of SIVs and their natural hosts did not occur in an otherwise pathogen-free environment, it is conceivable that the specific adaptations that allowed these species to avoid AIDS were influenced by the evolutionary pressure placed upon the immune system by other infectious agents. An alternative possibility is that a singular, relatively abrupt event in the distant past might have selected for a few animals that were resistant to disease progression and rapid death. In all of these scenarios, the underlying assumption is that SIV infections of natural hosts were originally pathogenic and thus exerted pressure on the host genome of the infected animal. Favorable characteristics in the gene pool of individuals surviving the infection were then selected for in successive generations. While this line of thinking is probably correct, it deserves some further qualification. A direct evolutionary pressure is certainly easy to imagine if the "ancestral" SIV infection of natural hosts manifested a tempo of disease similar to that observed in SIV-infected macaques (with progression to AIDS observed in most cases within 1-2 years of primary infection). It should be noted, however, that if the original and putatively pathogenic infections of African monkeys had a course of disease similar to that observed today in HIV-infected individuals (i.e., taking an average of 10 years to proceed from infection to death), it is hard to fathom a strong evolutionary pressure in animals with a 15-20 year lifespan in the wild that usually acquire infection as adults (75-78). In this perspective, it is possible to imagine that the original epidemics of SIV infection in African NHPs generated strong evolutionary pressure to avoid vertical transmission from mother to offspring, particularly if one considers (a) the likely deleterious effect of moving the "clock" of the infection back 4-5 years and (b) the fact that primate lentiviral infections appear to be more severe in newborns compared with adults $(79,80)$. As such, one may speculate that the genetic features of natural SIV hosts that underline their two main immunological features (i.e., low immune activation and decreased CCR5 expression on $\mathrm{CD} 4^{+} \mathrm{T}$ cells) may actually reflect evolutionary selection to reduce the incidence of vertical transmission from mother to offspring. Potential mechanisms whereby low levels of activated CD $4^{+} \mathrm{CCR} 5^{+} \mathrm{T}$ cells in fetuses and newborns may result in decreased frequency of vertical SIV infection include resistance to perinatal transmission due to contact with virus-infected maternal body fluids as well as protection from mucosal transmission caused by breastfeeding. The provocative corollary of this line of thinking is that these apparently protective features of natural SIV infection that we and others have observed in adult animals might be, at least to some extent, an epiphenomenon of evolutionary adaptations that were originally selected for as they had a positive impact on reducing or eliminating the risk of vertical transmission of SIVs.

In any event, the exact mechanisms by which evolutionary coadaptation has determined the exquisitely nonpathogenic phenotype of natural SIV infections remain to be defined - and it is certainly possible that different species of naturally adapted hosts may share some but not all these mechanisms, especially at the molecular level. A better definition of the evolutionary determinants of these nonpathogenic SIV infections will only be possible when experimental manipulation(s) of naturally adapted hosts will turn their nonpathogenic infection phenotype into a pathogenic one (or vice versa in the case of AIDS-susceptible species such as Asian macaques).

\section{Future directions and conclusions}

While significant progress has recently been made in our understanding of the main virological and immunological features of natural SIV infections, many important questions remain unanswered. Further studies are clearly warranted to better elucidate the mechanisms by which natural SIV hosts avoid disease progression. Such studies will undoubtedly provide important insights into the mechanisms of AIDS pathogenesis and will likely lead to the identification of new therapeutic targets $(81,82)$.

We have identified three research areas that, in our opinion, represent important topics for future studies. First and foremost, a strong effort should be made to elucidate the molecular mechanisms that underlie the dramatic differences in levels of immune activation during acute and chronic infections in natural and nonnatural hosts. The importance of this line of research is hard to overestimate since only the exact clarification of these mechanisms will identify targets for immunomodulatory strategies that hold promise of being both effective in reversing the course of disease and also safe in already immunosuppressed HIV-infected subjects. While targeting HIV-associated immune activation is, at least in principle, a promising therapeutic strategy for HIVinfected individuals, the fact that the mechanisms underlying this immune activation (and the mechanisms whereby natural hosts have attenuated it) are still poorly understood represents a major obstacle to the development of rational, targeted immunomodulatory interventions. This lack of basic knowledge is particularly worrisome when one considers that, ultimately, HIV infection results in a state of chronic immunodeficiency, in which the use of nonspecific immunosuppressive agents such as cyclosporine $\mathrm{A}$ may be ineffective (83) and even dangerous (84). A second major goal for future research efforts is to understand how low immune activation and $T$ cell turnover translate into a better preservation of $\mathrm{CD}^{+} \mathrm{T}$ cell counts. In this regard, the identification of differences in the regulation of the homeostasis of $\mathrm{CD}^{+} \mathrm{T}$ cells and their main subsets (Tn, Tcm, and Tem; see Figure 2) between AIDS-susceptible and AIDS-resistant naturally adapted hosts may have important therapeutic implications. Finally, we believe that it will be important to conduct studies aimed at identifying the 
mechanisms regulating the selectively reduced expression of CCR5 on $\mathrm{CD} 4^{+} \mathrm{T}$ cells of natural SIV hosts.

Solving the complex riddle of how natural SIV hosts avoid disease progression will require more time as well as the investment of a significant amount of scientific resources but will certainly represent a major step forward in the fight against AIDS.

\section{Acknowledgments}

The authors wish to thank Cristian Apetrei, Ann Chahroudi, Mark B. Feinberg, Amitinder Kaur, and Silvija I. Staprans for important discussions. The authors also wish to acknowledge members of the
Cleveland Immunopathogenesis Consortium for helpful discussions and sharing of data regarding the hypotheses discussed herein.

Address correspondence to: Guido Silvestri, Department of Pathology and Laboratory Medicine, University of Pennsylvania School of Medicine, 705 Stellar-Chance Laboratories, 422 Curie Blvd., Philadelphia, Pennsylvania 19104, USA. Phone: (215) 573-5363; Fax: (215) 573-5369; E-mail: gsilvest@mail.med.upenn.edu.

Donald L. Sodora's present address is: Seattle Biomedical Research Institute, Seattle, Washington, USA.
1. Hahn, B.H., Shaw, G.M., De Cock, K.M., and Sharp, P.M. 2000. AIDS as a zoonosis: scientific and public health implications. Science. 287:607-614.

2. VandeWoude, S., and Apetrei, C. 2006. Going wild: lessons from naturally occurring T-lymphotropic lentiviruses. Clin. Microbiol. Rev. 19:728-762.

3. Cichutek, K., and Norley, S. 1993. Lack of immune suppression in SIV-infected natural hosts. AIDS. 7:S25-S35.

4. Hirsch, V.M. 2004. What can natural infection of African monkeys with simian immunodeficiency virus tell us about the pathogenesis of AIDS? AIDS Rev. 6:40-53.

5. Chakrabarti, L.A. 2004. The paradox of simian immunodeficiency virus infection in sooty mangabeys: active viral replication without disease progression. Front. Biosci. 9:521-539.

6. Silvestri, G. 2005. Naturally SIV-infected sooty mangabeys: are we closer to understanding why they do not develop AIDS? J. Med. Primatol. 34:243-252.

7. Sharp, P.M., Shaw, G.M., and Hahn, B.H. 2005. Simian Immunodeficiency Virus infection of chimpanzee. J. Virol. 79:3891-3902.

8. Diop, O.M., et al. 2000. High levels of viral replication during primary simian immunodeficiency virus SIVagm infection are rapidly and strongly controlled in African green monkeys. J. Virol. 74:7538-7547.

9. Goldstein, S., et al. 2000. Wide range of viral load in healthy African green monkeys naturally infected with simian immunodeficiency virus. J. Virol. 74:11744-11753.

10. Broussard, S.R., et al. 2001. Simian immunodeficiency virus replicates to high levels in naturally infected African green monkeys without inducing immunologic or neurologic disease. J. Virol. 75:2262-2275.

11. Muller, M.C., and Barre-Sinoussi, F. 2003. SIVagm: genetic and biological features associated with replication. Front. Biosci. 8:d1170-d1185.

12. Pandrea, I., et al. 2003. High levels of SIVmnd-1 replication in chronically infected Mandrillus sphinx. Virology. 317:119-127.

13. Onanga, R., et al. 2006. Primary simian immunodeficiency virus SIVmnd-2 infection in mandrills (Mandrillus sphinx). J. Virol. 80:3301-3309.

14. Pandrea, I., et al. 2006. Simian immunodeficiency virus SIVagm.sab infection of Caribbean African green monkeys: a new model for the study of SIV pathogenesis in natural hosts. J. Virol. 80:4858-4867.

15. Rey-Cuille, M.A., et al. 1998. Simian immunodeficiency virus replicates to high levels in sooty mangabeys without inducing disease. J. Virol. 72:3872-3886.

16. Chakrabarti, L.A., et al. 2000. Normal T-cell turnover in sooty mangabeys harboring active simian immunodeficiency virus infection. J. Virol. 74:1209-1223.

17. Silvestri, G., et al. 2003. Nonpathogenic SIV infection of sooty mangabeys is characterized by limited bystander immunopathology despite chronic highlevel viremia. Immunity. 18:441-452.

18. Pandrea, I., et al. 2006. Simian immunodeficiency viruses replication dynamics in African non-human primate hosts: common patterns and species-specific differences. J. Med. Primatol. 35:194-201.

19. Sumpter, B., et al. 2007. Correlates of preserved $\mathrm{CD} 4(+) \mathrm{T}$ cell homeostasis during natural, nonpathogenic simian immunodeficiency virus infection of sooty mangabeys: implications for AIDS pathogenesis. J. Immunol. 178:1680-1691.

20. Apetrei, C., et al. 2007. Virus-subtype specific features of natural SIVsmm infection in sooty mangabeys. J. Virol. 81:7913-7923.

21. Mellors, J.W., et al. 1996. Prognosis in HIV-1 infection predicted by the quantity of virus in plasma. Science. 272:1167-1170.

22. Hirsch, V.M., et al. 1996. Patterns of viral replication correlate with outcome in simian immunodeficiency virus (SIV)-infected macaques: effect of prior immunization with a trivalent SIV vaccine in modified vaccinia virus Ankara. J. Virol. 70:3741-3752.

23. Staprans, S.I., et al. 1999. Simian immunodeficiency virus disease course is predicted by the extent of virus replication during primary infection. J. Virol. 73:4829-4839.

24. Norley, S., and Kurth, R. 2004. The role of the immune response during SIVagm infection of the African green monkey natural host. Front. Biosci. 9:550-564.

25. Dunham, R., et al. 2006. The AIDS resistance of naturally SIV-infected sooty mangabeys is independent of cellular immunity to the virus. Blood. 108:209-217.

26. Wang, Z., Metcalf, B., Ribeiro, R.M., McClure, H., and Kaur, A. 2006. Th-1-type cytotoxic CD8+ T-lymphocyte responses to simian immunodeficiency virus (SIV) are a consistent feature of natural SIV infection in sooty mangabeys. J. Virol. 80:2771-2783.

27. Barry, A.P., et al. 2007. Depletion of CD8+ cells in sooty mangabey monkeys naturally infected with SIV reveals limited role for immune control of virus replication in a natural host species. J. Immunol. 178:8002-8012.

28. Hazenberg, M.D., Hamann, D., Schuitemaker, H., and Miedema, F. 2000. T cell depletion in HIV-1 infection: how CD4+ T cells go out of stock. Nat Immunol. 1:285-289.

29. McCune, J.M. 2001. The dynamics of CD4+ T-cell depletion in HIV disease. Nature. 410:974-979.

30. Grossman, Z., et al. 2002. CD4+ T-cell depletion in HIV infection: are we closer to understanding the cause? Nat. Med. 8:319-323.

31. Silvestri, G., and Feinberg, M.B. 2003. Turnover of lymphocytes and conceptual paradigms in HIV infection. J. Clin. Invest. 112:821-824. doi:10.1172/ JCI200319799.

32. Kornfeld, C., et al. 2005. Antiinflammatory profiles during primary SIV infection in African green monkeys are associated with protection against AIDS. J. Clin. Invest. 115:1082-1091. doi:10.1172/ JCI200523006.

33. Gougeon, M.L., et al. 1997. Lack of chronic immune activation in HIV-infected chimpanzees correlates with the resistance of T cells to Fas/Apo-1 (CD95)- induced apoptosis and preservation of a $\mathrm{T}$ helper 1 phenotype. J. Immunol. 158:2964-2976.

34. Estes, J.D., et al. 2007. Simian immunodeficiency virus-induced lymphatic tissue fibrosis is mediated by transforming growth factor beta 1-positive regulatory $\mathrm{T}$ cells and begins in early infection. J. Infect. Dis. 195:551-561.

35. Estaquier, J., et al. 1994. Programmed cell death and AIDS: significance of T-cell apoptosis in pathogenic and nonpathogenic primate lentiviral infections. Proc. Natl. Acad. Sci. U. S. A. 91:9431-9435.

36. Kim, N., Dabrowska, A., Jenner, R.G., and Aldovini, A. 2007. Human and Simian immunodeficiency virus-mediated upregulation of the apoptotic factor TRAIL occurs in antigen presenting cells from AIDS-susceptible but not from AIDS-resistant species. J. Virol. 81:7584-7597.

37. Bostik, P., et al. 2001. Identification of protein kinases dysregulated in CD4(+) T cells in pathogenic versus apathogenic simian immunodeficiency virus infection. J. Virol. 75:11298-11306.

38. Bostik, P., Dodd, G.L., Villinger, F., Mayne, A.E., and Ansari, A.A. 2004. Dysregulation of the pololike kinase pathway in CD4+ T cells is characteristic of pathogenic simian immunodeficiency virus infection. J. Virol. 78:1464-1472.

39. Paiardini, M., et al. 2006. Perturbations of cell cycle control in T cells contribute to the different outcomes of simian immunodeficiency virus infection in rhesus macaques and sooty mangabeys. J. Virol. 80:634-642.

40. Muthukumar, A., et al. 2005. Timely triggering of homeostatic mechanisms involved in the regulation of T-cell levels in SIVsm-infected sooty mangabeys. Blood. 106:3839-3845.

41. Silvestri, G., et al. 2005. Divergent host responses during primary simian immunodeficiency virus SIVsm infection of natural sooty mangabey and nonnatural rhesus macaque hosts. J. Virol. 79:4043-4054.

42. Gordon, S.N., et al. 2007. Severe depletion of mucosal CD4+ T-cells in AIDS-free SIV-infected sooty mangabeys. J. Immunol. 179:3026-3034.

43. Kaur, A., et al. 2001. Emergence of cytotoxic T lymphocyte escape mutations in nonpathogenic simian immunodeficiency virus infection. Eur. J. Immunol. 31:3207-3217.

44. Liu, Z., et al. 1997. Elevated CD38 antigen expression on CD8+ T cells is a stronger marker for the risk of chronic HIV disease progression to AIDS and death in the Multicenter AIDS Cohort Study than CD4+ cell count, soluble immune activation markers, or combinations of HLA-DR and CD38 expression. J. Acquir. Immune Defic. Syndr. Hum. Retrovirol. 16:83-92.

45. Giorgi, J.V., et al. 1999. Shorter survival in advanced human immunodeficiency virus type 1 infection is more closely associated with $\mathrm{T}$ lymphocyte activation than with plasma virus burden or virus chemokine coreceptor usage. J. Infect. Dis. 179:859-870.

46. Giorgi, J.V., et al. 2002. Multicenter AIDS Cohort Study. Predictive value of immunologic and virologic markers after long or short duration of HIV-1 infection. J. Acquir. Immune. Defic. Syndr. 29:346-355. 
47. Pereira, L.E., et al. 2007. Simian Immunodeficiency Virus (SIV) infection influences the level and function of regulatory $\mathrm{T}$ cells in SIV-infected rhesus macaques but not SIV-Infected sooty mangabeys. J. Virol. 81:4445-4456.

48. Schindler, M., et al. 2006. Nef-mediated suppression of $\mathrm{T}$ cell activation was lost in a lentiviral lineage that gave rise to HIV-1. Cell. 125:1055-1067.

49. Guadalupe, M., et al. 2003. Severe CD4+ T-cell depletion in gut lymphoid tissue during primary human immunodeficiency virus type 1 infection and substantial delay in restoration following highly active antiretroviral therapy. J. Virol. 77:11708-11717.

50. Picker, L.J., et al. 2004. Insufficient production and tissue delivery of CD4+ memory $\mathrm{T}$ cells in rapidly progressive simian immunodeficiency virus infection. J. Exp. Med. 200:1299-1314.

51. Brenchley, J.M., et al. 2004. CD4+ T cell depletion during all stages of HIV disease occurs predominantly in the gastrointestinal tract. J. Exp. Med. 200:749-759.

52. Mehandru, S., et al. 2004. Primary HIV-1 infection is associated with preferential depletion of CD4+ T lymphocytes from effector sites in the gastrointestinal tract. J. Exp. Med. 200:761-770.

53. Mattapallil, J.J., et al. 2005. Massive infection and loss of memory CD4+ T cells in multiple tissues during acute SIV infection. Nature. 434:1093-1097.

54. Li, Q., et al. 2005. Peak SIV replication in resting memory CD4+ T cells depletes gut lamina propria CD4+ T cells. Nature. 434:1148-1152.

55. Picker, L.J., and Watkins, D.I. 2005. HIV pathogenesis: the first cut is the deepest. Nat. Immunol. 6:430-432.

56. Veazey, R.S., and Lackner, A.A. 2005. HIV swiftly guts the immune system. Nat. Med. 11:469-470.

57. Haase, A.T. 2005. Perils at mucosal front lines for HIV and SIV and their hosts. Nat. Rev. Immunol. 5:783-789.

58. Johnson, R.P., and Kaur, A. 2005. HIV: viral blitzkrieg. Nature. 434:1080-1081.

59. Brenchley, J.M., Price, D.A., and Douek, D.C. 2006. HIV disease: fallout from a mucosal catastrophe? Nat. Immunol. 7:235-239.

60. Brenchley, J.M., et al. 2006. Microbial translocation is a cause of systemic immune activation in chronic HIV infection. Nat. Med. 12:1365-1371.
61. Pandrea, I., et al. 2007. Acute loss of intestinal CD4 ${ }^{+}$ T-cells is not predictive of SIV virulence. J. Immunol. 179:3035-3046.

62. Milush, J.M., et al. 2007. Virally-induced CD4+ T cell depletion is not sufficient to induce AIDS in a natural host. J. Immunol. 179: 3026-3034.

63. Chen, Z., Gettie, A., Ho, D.D., and Marx, P.A. 1998. Primary SIVsm isolates use the CCR5 coreceptor from sooty mangabeys naturally infected in west Africa: a comparison of coreceptor usage of primary SIVsm, HIV-2, and SIVmac. Virology. 246:113-124.

64. Gautam, R., et al. 2007. In vitro characterization of primary SIVsmm isolates belonging to different lineages. In vitro growth on rhesus macaque cells is not predictive for in vivo replication in rhesus macaques. Virology. 362:257-270.

65. Connor, R.I., Sheridan, K.E., Ceradini, D., Choe, S., and Landau, N.R. 1997. Change in coreceptor use coreceptor use correlates with disease progression in HIV-1--infected individuals. J. Exp. Med. 185:621-628

66. Scarlatti, G., et al. 1997. In vivo evolution of HIV-1 co-receptor usage and sensitivity to chemokinemediated suppression. Nat. Med. 3:1259-1265.

67. Lane, H.C., et al. 1983. Abnormalities of B-cell activation and immunoregulation in patients with the acquired immunodeficiency syndrome. N. Engl. J. Med. 309:453-458.

68. Braun, D.P., et al. 1988. Monocyte functional studies in asymptomatic, human immunodeficiency disease virus (HIV)-infected individuals. J. Clin. Immunol. 8:486-494.

69. Crowe, S.M., and Kornbluth, R.S. 1994. Overview of HIV interactions with macrophages and dendritic cells: the other infection in AIDS. J. Leukoc. Biol. 56:215-217.

70. Martinez-Maza, O., Crabb, E., Mitsuyasu, R.T., Fahey, J.L., and Giorgi, J.V. 1987. Infection with the human immunodeficiency virus (HIV) is associated with an in vivo increase in B lymphocyte activation and immaturity. J. Immunol. 138:3720-3724.

71. Poccia, F., et al. 1996. Peripheral V gamma 9/V delta $2 \mathrm{~T}$ cell deletion and anergy to nonpeptidic mycobacterial antigens in asymptomatic HIV-1-infected persons. J. Immunol. 157:449-461.

72. Zhou, D., et al. 2003. Inhibition of adaptive Vgamma2Vdelta2+ T-cell responses during active mycobacte- rial coinfection of simian immunodeficiency virus SIVmac-infected monkeys. J. Virol. 77:2998-3006.

73. Pandrea, I., et al. 2007. Paucity of CD4+CCR5+ T cells is a typical feature of natural SIV hosts. Blood. 109:1069-1076.

74. Seder, R.A., and Ahmed, R. 2003. Similarities and differences in $\mathrm{CD} 4+$ and CD8+ effector and memory T cell generation. Nat. Immunol. 4:835-842.

75. Fultz, P.N., et al. 1990. Prevalence of natural infection with simian immunodeficiency virus and simian T-cell leukemia virus type I in a breeding colony of sooty mangabey monkeys. AIDS. 4:619-625.

76. Otsyula, M.G., et al. 1995. Apparent lack of vertical transmission of simian immunodeficiency virus (SIV) in naturally infected African green monkeys, Cercopithecus aethiops. Ann. Trop. Med. Parasitol. 89:573-576.

77. Nerrienet, E., et al. 1998. Phylogenetic analysis of SIV and STLV type I in mandrills (Mandrillus sphinx): indications that intracolony transmissions are predominantly the result of male-to-male aggressive contacts. AIDS Res. Hum. Retroviruses. 14:785-796.

78. Ellis, B.R., et al. 2004. Seroprevalence of simian immunodeficiency virus in wild and captive born Sykes' monkeys (Cercopithecus mitis) in Kenya. Retrovirology. 1:34.

79. Pizzo, P.A., Eddy, J., and Faloon, J. 1988. Acquired immune deficiency syndrome in children. Current problems and therapeutic considerations. Am.J. Med. 85:195-202.

80. Marthas, M.L., et al. 1995. Viral factors determine progression to AIDS in simian immunodeficiency virus-infected newborn rhesus macaques. J. Virol. 69:4198-4205.

81. Stevenson, M. 2003. HIV-1 pathogenesis. Nat. Med. 9:853-860.

82. Stebbing, J., Gazzard, B., and Douek, D.C. 2004. Where does HIV live? N. Engl. J. Med. 350:1872-1880.

83. Lederman, M.M., et al. 2006. Cyclosporin A provides no sustained immunologic benefit to persons with chronic HIV-1 infection starting suppressive antiretroviral therapy: results of a randomized, controlled trial of the AIDS clinical trials group A5138. J. Infect. Dis. 194:1677-1685.

84. Phillips, A., et al. 1989. Cyclosporine-induced deterioration in patients with AIDS. CMAJ. 140:1456-1460. 